\title{
Emotional states of different obesity phenotypes: a sex-specific study in a west- Asian population
}

Fahimeh Mehrabi ${ }^{1}$, Parisa Amiri ${ }^{*}$ (D), Leila Cheraghi ${ }^{1,2}$, Ali Kheradmand ${ }^{3}$, Farhad Hosseinpanah ${ }^{4}$ and Fereidoun Azizi ${ }^{5}$

\begin{abstract}
Background: The present study aimed to investigate the associations of obesity phenotypes with depression, anxiety, and stress symptoms among adults in the Tehran Lipid and Glucose Study (TLGS).

Methods: Depression, anxiety, and stress levels of participants from the TLGS were examined among different obesity phenotypes in this cross-sectional study.

Obesity was defined as body mass index (BMI) $\geq 30 \mathrm{~kg} / \mathrm{m}^{2}$, and metabolically unhealthy status based on having metabolic syndrome (MetS) or type 2 diabetes. Four obesity phenotypes were defined: 1) Metabolically Healthy Non-Obese (MHNO), 2) Metabolically Healthy Obese (MHO) 3) Metabolically Unhealthy Non-Obese (MUNO), and 4) Metabolically Unhealthy Obese (MUO). Emotional states of different obesity phenotypes were assessed by the Persian version of depression, anxiety, and stress scale-21 items (DASS-21). Ordinal logistic regression analysis was used to compare sex-specific odds ratios of depression, anxiety, and stress in different obesity phenotypes.

Results: The mean age of 2469 men and women was $46.2 \pm 15.9$ and $45.6 \pm 14.7$, respectively. In total, women were more likely to experience higher levels of depression (30.5\%), anxiety (44.2\%), and stress (43.5\%) symptoms compared to men. After adjusting for potential confounders, compared to MHNO men, the odds of experiencing higher anxiety levels were significantly greater in metabolically unhealthy men whether they were obese (OR: 1.78 , 95\% Cl: $1.25-2.54 ; P=<0.001$ ) or non-obese (OR: 1.61, 95\% Cl: 1.17-2.21; $P=<0.001$ ), and also in MUO women (OR: $1.73,95 \% \mathrm{Cl}: 1.28-2.34 ; P=<0.001)$ compared to MHNO women. Moreover, the odds of experiencing higher stress levels were significantly greater in MUNO men (OR: 1.40,95\% Cl: 1.02-1.90; $P=0.04)$ compared to MHNO men and in $\mathrm{MUO}$ women (OR: 1.45, 95\% Cl: 1.07-1.96; $P=0.02)$ compared to MHNO women. No difference in depression levels was observed in either sex.
\end{abstract}

Conclusions: Our results showed that men and women with various obesity phenotypes experienced different anxiety and stress levels. While MUO women and all metabolically unhealthy men experienced more anxiety and stress levels than MHNO individuals, none of the obesity phenotypes were associated with depression. These findings provide insight into recognizing the psychological consequences of different phenotypes of obesity in both sexes and utilizing future health promotion planning.

Keywords: Metabolic syndrome, Obesity, Depression, Anxiety, Stress, Metabolically healthy obesity

\footnotetext{
*Correspondence: amiri@endocrine.ac.ir; parisaamiri@yahoo.com

${ }^{1}$ Research Center for Social Determinants of Health, Research Institute for

Endocrine Sciences, Shahid Beheshti University of Medical Sciences, P.O.Box:

19395-4763, Tehran, Iran

Full list of author information is available at the end of the article
}

\section{$\triangle B M C$}

(c) The Author(s). 2021 Open Access This article is licensed under a Creative Commons Attribution 4.0 International License, which permits use, sharing, adaptation, distribution and reproduction in any medium or format, as long as you give appropriate credit to the original author(s) and the source, provide a link to the Creative Commons licence, and indicate if changes were made. The images or other third party material in this article are included in the article's Creative Commons licence, unless indicated otherwise in a credit line to the material. If material is not included in the article's Creative Commons licence and your intended use is not permitted by statutory regulation or exceeds the permitted use, you will need to obtain permission directly from the copyright holder. To view a copy of this licence, visit http://creativecommons.org/licenses/by/4.0/ The Creative Commons Public Domain Dedication waiver (http://creativecommons.org/publicdomain/zero/1.0/) applies to the data made available in this article, unless otherwise stated in a credit line to the data. 


\section{Background}

Obesity is a common health issue that has tripled over the last decades [1]. Along with the global ascending rate of excessive weight gain, a range of $18.5-25 \%$ as an average prevalence for obesity among the Iranian adult population has also been reported [2]. Obesity has been identified as the leading cause of chronic disorders, such as cardiovascular diseases (CVDs), diabetes mellitus, cancers, and premature death [3]. One of the complications of obesity is related to its frequent incidence with metabolic syndrome (MetS). According to some studies, measuring only body mass index (BMI) in evaluating the outcomes of obesity is misleading since individuals' metabolic status is an equally essential determinant as BMI [4]. In this regard, obesity phenotypes can be represented as combinations of BMI and metabolic health components, leading to different health outcomes [5].

There are fewer investigations about the mental health outcomes of obesity and MetS than physical comorbidities. In the realm of psychological consequences, it has been indicated that metabolic disturbances are partly responsible for increased mortality in schizophrenic and bipolar patients [6]. Yet, there is no certain consensus about the predisposing impacts of obesity and MetS on common mental health outcomes such as depression, anxiety, and stress symptoms. These conditions, which are more common in women than men [7], affect individuals' moods or feelings, decline productivity, and cause a tremendous economic burden $[8,9]$. The prevalence of depression (44\%), anxiety (42\%), and stress (40\%) symptoms among the general population in Iran is surprisingly high, with a higher rate of incidence in women [10]. Due to the sex differences in depression, anxiety, and stress symptoms, in addition to specific physical and socio-environmental determinants in men and women, it seems essential to conduct a sex-specific study to recognize factors affecting them, such as different obesity phenotypes.

Although Individuals with obesity are believed to have more depression and anxiety at clinical and subclinical levels, supported by a large body of evidence [11, 12], some studies have not found any relationship between obesity and mental health outcomes [13-15]. Even others have established hypothesis emphasizing that higher BMI could lead to fewer mental health issues, including depression and anxiety in different populations [16-18]. In terms of MetS, similar conflicting results have been published on depression and anxiety symptoms associated with unfavorable metabolic profiles [1922]. The previous studies also examined the bidirectional relationship and the existence of a vicious cycle between obesity and stress [23], which has been considered a risk factor for some metabolic syndrome parameters [24]. However, a recent meta-analysis on four qualified studies indicated no relationship between MetS and stress levels in the adult population [24].

The remarkable point is that despite the frequent concurrence of obesity and MetS, most of the prior studies have not considered the heterogeneity of obesity phenotypes. Only a few studies were conducted to assess whether or not being a metabolically healthy obese (MHO) phenotype is a psychologically benign situation compared to other phenotypes, and most of them only considered depression as an outcome [25-29]. Examining the effects of two common somatic diseases on mental health conditions could shed light on one aspect of these multifactorial disorders. The current study aimed to investigate the association of obesity phenotypes with depression, anxiety, and stress symptoms in adult participants of the Tehran Lipid and Glucose Study (TLGS). The obtained results could provide a comprehensive view regarding the emotional states of a large general population in West Asia.

\section{Methods \\ Study design and participants}

This study was conducted in the framework of the TLGS. The TLGS includes two major junctures: The first juncture was the 1st phase, a cross-sectional study designed to determine the prevalence of noncommunicable diseases (NCDs) risk factors executed from 1999 to 2001. A total of 15,005 individuals aged $\geq$ three who were residents of district 13 of Tehran were recruited in the study. The second juncture includes five follow-up phases that have been implemented from 2002 to 2019 every 3 years. More study details have been reported previously [30].

In the current study, from all the individuals who participated in the TLGS during the 2016-2019 (6th phase), 2728 participants aged $\geq 20$ years with complete data on depression, anxiety, and stress were recruited. After excluding those with missing data on BMI, MetS components, or covariates (sociodemographic factors, smoking status, and level of physical activity) $(n=259)$, the final data of 2469 adults (1158 men and 1311 women) were analyzed. This study was approved by the ethics committee of the Research Institute for Endocrine Sciences, Shahid Beheshti University of Medical Sciences. All the participants signed the written informed consent before data collection.

\section{Definition and measurements Biochemical measurements}

The blood sample was taken from the participants after a 12-14 h overnight fast by trained personnel in the data collection center of the TLGS. All blood samples of 
fasting blood sugar (FBS) and serum lipids (total cholesterol (TC), high-density lipoprotein cholesterol (HDLC), and triglycerides (TG)) were analyzed in the TLGS research laboratory on the same day. Additional information has been previously published about the biochemical [31].

\section{Clinical and anthropometric measurements}

Trained personnel measured the weight, height, and waist circumferences of participants while they wore light clothes and were barefoot. The weight was measured using an electronic digital scale that its accuracy was up to $100 \mathrm{~g}$. The height was examined in $\mathrm{cm}$, while participants were standing normally using a tape meter stadiometer. Waist circumference was measured via an unstretched measuring tape and recorded to the nearest $0.1 \mathrm{~cm}$. The blood pressure was measured after a $15-\mathrm{min}$ rest period in the seated position twice by qualified physicians via a standard mercury sphygmomanometer; the average of two measurements was considered for analysis.

\section{MetS and type 2 diabetes}

MetS was defined as having any three of the following abnormalities: 1) ethnic-based abdominal obesity, which was defined as waist circumference $\geq 90 \mathrm{~cm}$ for men and women [32]; 2) HDL-cholesterol $<40 \mathrm{mg} / \mathrm{dl}$ in men or $50 \mathrm{mg} / \mathrm{dl}$ in women; 3) triglyceride $\geq 150 \mathrm{mg} / \mathrm{dl}$; 4) glucose $\mathrm{FBS} \geq 126 \mathrm{mg} / \mathrm{dl}$ or known treatment for diabetes; 5) blood pressure $\geq 130 / 85 \mathrm{mmHg}$ or use of antihypertensive drugs [33]. Type 2 diabetes was defined as fasting blood sugar $\mathrm{FBS} \geq 126 \mathrm{mg} / \mathrm{dl}$ or 2 -h post-load glucose $\geq 200 \mathrm{mg} / \mathrm{dl}$ or taking medication for diagnosed diabetes. Obesity was defined as BMI $\geq 30 \mathrm{~kg} / \mathrm{m}^{2}$.

\section{Obesity phenotypes}

The participants were categorized into four obesity phenotypes: 1) Metabolically healthy non-obese (MHNO), 2) Metabolically healthy obese (MHO), 3) Metabolically unhealthy non-obese (MUNO), and 4) Metabolically unhealthy obese (MUO). Metabolically unhealthy status was defined as having MetS or diabetes type 2, according to the Joint Interim Statement (JIS) and the American Diabetes Association (ADA), respectively.

\section{Sociodemographic characteristics}

The participants' age, sex, marital status, job status, and educational level were assessed via a pretest questionnaire. Participants' educational level was defined as 1) Primary: including people with less than a high school diploma; 2) Secondary: including people with a high school diploma; and 3) Higher: including people with a college degree or higher.

\section{Smoking and physical activity status}

The smoking habit of participants was classified into two groups: 1) smokers (daily and occasionally smokers) and 2) non-smokers (ex-smokers or never smokers). Physical activity levels were evaluated by the validated Iranian version of the Modifiable Activity Questionnaire (MAQ). The frequency and duration of each leisuretime and work physical activity (standing, housework, and work activities more intense than standing) were calculated as hour/week. Then they were multiplied by the weight and the metabolic equivalent task (MET) of the particular act to calculate the energy expenditure for each domain. Total physical activity was calculated by adding each domain's energy expenditure and was categorized into three groups of low $(<600)$, moderate (600-3000), and high ( $\geq 3000)$ physical activity [34].

\section{Depression, anxiety, and stress}

The Persian version of depression, anxiety, and stress scale-21 items (DASS-21) was used to assess emotional distress among participants. The psychometric properties of the Persian version of DASS-21 have been previously studied among the Iranian population, and its reliability and validity were approved [35]. DASS-21 is a self-report questionnaire, including three scales, and each scale was composed of seven items divided into subscales with similar content. Examples of items in each subscale are "I couldn't seem to experience any positive feeling at all" for depression; "I experienced trembling (e.g., in the hands)" for anxiety; and "I felt that I was using a lot of nervous energy" for stress. The participants completed this questionnaire by rating each item to reflect their emotional experiences over the past week from 0 (did not apply to me at all) to 3 (applied to me very much). Depression, anxiety, and stress were treated as ordinal variables in the current study. The cut-off scores for conventional severity labels were used as follows: 1) Depression: normal: 0-9, mild: 10-13, moderate: $14-20$, and severe: +21 ; 2 ) Anxiety: normal: $0-7$, mild: $8-9$, moderate: $10-14$, and severe: $+15 ; 3$ ) Stress: normal: $0-14$, mild: $15-18$, moderate: $19-25$, and severe: +26. According to the DASS-21 scoring structure, each scale in this questionnaire was multiplied by two, so the highest score for each scale was 42 [36].

\section{Statistical analysis}

Continuous variables were expressed as mean \pm standard deviation (SD), and categorical variables were expressed as frequency (percentage). The continuous and categorical variables among different obesity phenotypes were compared via the one-way ANOVA and Chi-square test, respectively. To show an overview and a general comparison, mean scores of depression, anxiety, and stress were compared across different obesity phenotypes via 
the analysis of covariance and age, marital status, education, job status, smoking status, and level of physical activity were considered as adjustments. Ordinal logistic regression was used to explore the associations across obesity phenotypes and ordinal outcomes of depression, anxiety, and stress. Sex-specific odds ratios (ORs) with 95\% confidence intervals were calculated and reported for men and women separately; model 1 was unadjusted, while model 2 was adjusted for age, marital status (Ref.: Married), education (Ref.: Higher), job status (Ref.: Employed), smoking status (Ref.: Non-smoker), and level of physical activity (Ref: High). All tests were two-sided, and a $p$-value of less than 0.05 was considered statistically significant. After controlling the false discovery rate $(\mathrm{FDR}=0.2)$ by the method of Benjamini-Hochberg [37], all $p$-values under 0.04 remained significant. Statistical analysis was conducted using the IBM SPSS 24 (SPSS Inc., Chicago, IL, USA).

\section{Results}

The mean age of 2469 men and women was $46.2 \pm 15.9$ and $45.6 \pm 14.7$ years, respectively. The distribution of sociodemographic factors, smoking status, and level of physical activity among study groups are illustrated in Table 1 . The prevalence of obesity phenotypes is $45.9 \%$ (MHNO) group (45.9\%), 23.6\% (MUNO), 20.9\% (MUO), $9.6 \%$ (MHO). The majority of participants in all groups were married $(79.9 \%$ men and $75.1 \%$ women). Most metabolically unhealthy male subjects had a college degree ( $40 \%$ non-obese and $43.4 \%$ obese), while most metabolically unhealthy women had a high school diploma or less (45.6\% non-obese and $51.1 \%$ obese). Most men across all phenotypes (74\%) were employed, while most women $(71.5 \%)$ were unemployed or identified as housewives. The prevalence of type 2 diabetes among MUNO and MUO phenotypes was 20 and 16.6\% among men, likewise 33.8 and $25.1 \%$ among women. The mean BMI of participants was $27.79 \pm 4.85$. The descriptive statistics of BMI and MetS components in men and women are represented in Table 1-Additional file 1.

Table 2 illustrates the distribution of depression, anxiety, and stress levels among phenotypes for men and women. In total, women were more likely to experience higher levels of depression (30.5\%), anxiety (44.2\%), and stress $(43.5 \%)$, and the number of severe depression, anxiety, and stress in women $(7.3,15.5$, and $16.6 \%)$ was higher than men $(4.7,8.2$, and $9.5 \%)$. The highest and lowest frequency in the entire sample were MHNO women with normal depression levels and MHO men with moderate and severe depression, respectively. The mean scores of depression, anxiety, and stress were compared among different obesity phenotypes after adjusting for age, marital status, level of education, job status, smoking status, and level of physical activity and were illustrated in (Fig. 1). The results showed that mean anxiety scores in men and mean anxiety and stress scores in women were significantly different among obesity phenotypes ( $p=0.044, p=0.02$, and $p=0.022$, respectively). However, there was no significant difference in the depression scale in both sexes.

Table 3 shows the odds ratios $(95 \% \mathrm{CI})$ of reporting higher levels of depression, anxiety, and stress for different obesity phenotypes for men and women separately. After adjusting for potential confounders, including age, marital status, level of education, job status, smoking status, and level of physical activity, the odds of experiencing higher levels of anxiety were significantly greater in MUO (OR: 1.78, 95\% CI: 1.25, 2.54; $p=<0.001$ ) and MUNO men (OR: 1.61, 95\% CI: 1.17, 2.21; $p=<0.001$ ) compared to MHNO men, and also in MUO women (OR: $1.73,95 \%$ CI: $1.28,2.34 ; p=<0.001$ ) compared to MHNO women. Moreover, the odds of experiencing higher stress levels were significantly greater in MUNO men (OR: 1.40, 95\% CI: 1.02, 1.90; $p=0.04$ ) and in MUO women (OR: 1.45, 95\% CI: 1.07, 1.96; $p=0.02$ ) compared to MHNO men and women, respectively. The difference in having higher depression levels was observed in MUO women before adjustment (OR: 1.39, 95\% CI: $1.04,1.84 ; p=0.02$ ), but no difference was observed after adjustments in both sexes.

\section{Discussion}

The present study was one of the first attempts to investigate the relation of obesity phenotypes with emotional distress among men and women in Tehran. In total, the current results indicated that women across all phenotypes were more likely to experience depression, anxiety, and stress symptoms compared to men. More stratified analysis based on weight and metabolic status revealed an increased risk of anxiety and stress among MUO women compared to their MHNO counterparts. However, corresponding results for men showed that regardless of weight status, metabolic conditions were associated with higher anxiety and stress levels. Interestingly, obesity phenotypes were not related to depression in either sex.

The current results indicated that women were more likely to report experiencing negative mental health symptoms, mainly in the form of anxiety and stress. Consistent with our findings, sex differences and higher prevalence of mood and anxiety disorders among women have been addressed in Iran [10] and other nations as well [38]. Apart from genetic and other biological factors like different hormonal fluctuations in women [39], some essential psychosocial determinants, including more extended rumination and brooding [40], shame, interpersonal stressors, and experienced violence in women, are considered the reasons for this higher 
Table 1 Distribution of participants'characteristics in different obesity phenotypes $(n=2469)$

\begin{tabular}{|c|c|c|c|c|c|c|c|}
\hline \multirow[t]{2}{*}{ Variables } & & \multirow[t]{2}{*}{ Total } & \multicolumn{2}{|c|}{ Metabolically healthy } & \multicolumn{2}{|c|}{ Metabolically unhealthy } & \multirow[t]{2}{*}{$P$-value } \\
\hline & & & $\begin{array}{l}\text { Non-obese } \\
n=1133 \\
(45.9)\end{array}$ & $\begin{array}{l}\text { Obese } \\
n=237 \\
(9.6)\end{array}$ & $\begin{array}{l}\text { Non-obese } \\
n=583 \\
(23.6) \\
\end{array}$ & $\begin{array}{l}\text { Obese } \\
n=516(20.9)\end{array}$ & \\
\hline \multirow[t]{18}{*}{ Men } & Age (year) & $46.2 \pm 15.9$ & $41.36 \pm 15.8$ & $48.5 \pm 12.9$ & $54.1 \pm 15.2$ & $40.9 \pm 11.6$ & $<0.001$ \\
\hline & Marital status n(\%) & & & & & & $<0.001$ \\
\hline & Single & $233(20.1)$ & 168(32.8) & 16(18.6) & $29(8.2)$ & $20(9.8)$ & \\
\hline & Married & $925(79.9)$ & $344(67.2)$ & 70(81.4) & 326(91.8) & $185(90.2)$ & \\
\hline & Level of education $n(\%)$ & & & & & & $<0.001$ \\
\hline & Primary & 219 (18.9) & $62(12.1)$ & $14(16.3)$ & $101(28.5)$ & $42(20.5)$ & \\
\hline & Secondary & $481(41.5)$ & $216(42.2)$ & $34(39.5)$ & $142(40.0)$ & $89(43.4)$ & \\
\hline & Higher & 458 (39.6) & $234(45.7)$ & $38(44.2)$ & $112(31.5)$ & $74(36.1)$ & \\
\hline & Job status n(\%) & & & & & & $<0.001$ \\
\hline & Unemployed, but had other sources of income & $212(18.3)$ & $59(11.5)$ & $4(4.7)$ & $118(33.2)$ & $31(15.1 .8)$ & \\
\hline & Unemployed/housewife & $89(7.7)$ & $65(12.7)$ & $4(4.7)$ & $13(3.7)$ & $7(3.4)$ & \\
\hline & Employed & $857(74.0)$ & $388(75.8)$ & $78(90.7)$ & $224(63.1)$ & $167(81.5)$ & \\
\hline & Smoking status $\mathrm{n}(\%)$ & & & & & & 0.21 \\
\hline & Smoker & $498(43.0)$ & $213(41.6)$ & $33(38.4)$ & $151(42.5)$ & $101(49.3)$ & \\
\hline & Non-smoker & $660(57.0)$ & $299(58.4)$ & $53(61.6)$ & $204(57.5)$ & $104(50.7)$ & \\
\hline & Level of Physical activity $\mathrm{n}(\%)$ & & & & & & 0.02 \\
\hline & Low & $451(38.9)$ & $174(34.0)$ & $36(41.9)$ & $150(42.3)$ & $91(44.4)$ & \\
\hline & Moderate or High & $707(61.1)$ & $338(66.0)$ & $50(58.1)$ & $205(57.7)$ & $114(55.6)$ & \\
\hline \multirow[t]{19}{*}{ Women } & Age (year) & $45.6 \pm 14.7$ & $38.3 \pm 12.3$ & $46.2 \pm 12.2$ & $55.5 \pm 13.2$ & $48.5 \pm 12.9$ & $<0.001$ \\
\hline & Marital status n(\%) & & & & & & $<0.001$ \\
\hline & Single & $184(14.0)$ & $148(23.8)$ & $13(8.6)$ & $12(5.3)$ & $11(3.5)$ & \\
\hline & Divorced/Widowed & $142(10.8)$ & $28(4.5)$ & $17(11.3)$ & $39(17.1)$ & $58(18.6)$ & \\
\hline & Married & $985(75.1)$ & $445(71.7)$ & $121(80.1)$ & $177(77.6)$ & $242(77.8)$ & \\
\hline & Level of education $\mathrm{n}(\%)$ & & & & & & $<0.001$ \\
\hline & Primary & $372(28.4)$ & $59(9.5)$ & $50(33.1)$ & $104(45.6)$ & $159(51.1)$ & \\
\hline & Secondary & $525(40.0)$ & $273(44.0)$ & $60(39.7)$ & $84(36.8)$ & $108(34.7)$ & \\
\hline & Higher & $414(31.6)$ & $289(46.5)$ & $41(27.2)$ & $40(17.5)$ & $44(14.1)$ & \\
\hline & Job status n(\%) & & & & & & $<0.001$ \\
\hline & Unemployed, but had other sources of income & $132(10.1)$ & $26(4.2)$ & $12(7.9)$ & $42(18.4)$ & $52(16.7)$ & \\
\hline & Unemployed/housewife & $937(71.5)$ & $431(69.4)$ & $112(74.2)$ & $153(67.1)$ & $241(77.5)$ & \\
\hline & Employed & $242(18.5)$ & $164(26.4)$ & $27(17.9)$ & $33(14.5)$ & $18(5.8)$ & \\
\hline & Smoking status $\mathrm{n}(\%)$ & & & & & & 0.38 \\
\hline & Smoker & $69(5.3)$ & $38(6.1)$ & $4(2.6)$ & $11(4.8)$ & $16(5.1)$ & \\
\hline & Non-smoker & $1242(94.7)$ & $583(93.9)$ & $147(97.4)$ & $217(95.2)$ & $295(94.9)$ & \\
\hline & Level of Physical activity $\mathrm{n}(\%)$ & & & & & & 0.71 \\
\hline & Low & $383(29.2)$ & $173(27.9)$ & $44(29.1)$ & $68(29.8)$ & $98(31.5)$ & \\
\hline & Moderate or High & $928(70.8)$ & $448(72.1)$ & $107(70.9)$ & 160 (70.2) & $213(68.5)$ & \\
\hline
\end{tabular}

Data are presented as mean $\pm S D$ and frequency (\%). Variables were compared via the one-way ANOVA and Chi-square test

prevalence worldwide. Also, gender inequality, traditional gender roles, and sex-based discrimination are recognized as influential cultural factors [41]. In transitional societies, including most Middle-Eastern countries, all developments in the community's economic and educational structure in recent decades have 
Table 2 Sex-specific levels of depression, anxiety, and stress in different Obesity phenotypes $(n=2469)$

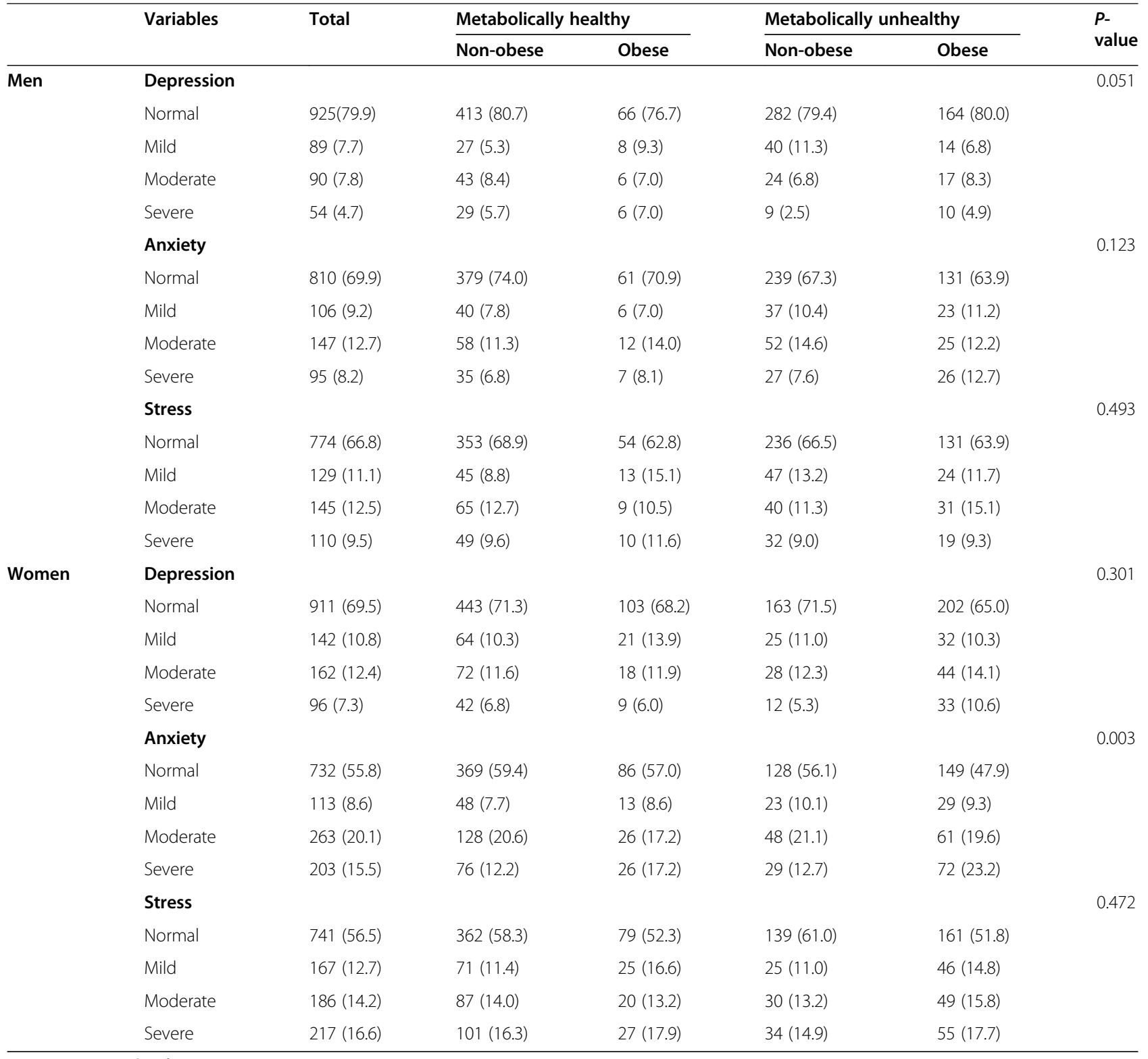

been accompanied by the multiplicity of women's expected roles, which could complicate the underlying causes of mentioned mental health outcomes [42].

There were significant relationships among obesity phenotypes with anxiety and stress in both sexes in the current study. The previous studies focused mainly on depression as a mental health construct regarding obesity phenotypes. On the other hand, using different measurement tools for assessing mental health outcomes, investigating separate effects of obesity and cardiovascular risk factors, and various cultural contexts make the comparison difficult. To the best of our knowledge, only one study among a middle-aged Irish population revealed a higher risk of anxiety in MUO individuals than their MHNO counterparts [26]. Other studies focused on weight and metabolic health status separately. In this regard, a meta-analysis reported a more frequent incidence of anxiety among individuals with obesity compared to those without obesity [12]. Conversely, some evidence showed the lack of association between MetS and anxiety among Japanese men [19]. Regarding stress, the findings of a recent meta-analysis on four studies indicated no association between MetS and stress [24]. In the current study, higher levels of anxiety and stress were simultaneously observed in the same definite phenotypes in both sexes. This is in line with previous findings indicating chronic stress leads to anxiety, and anxiety makes individuals vulnerable to stress [43]. 


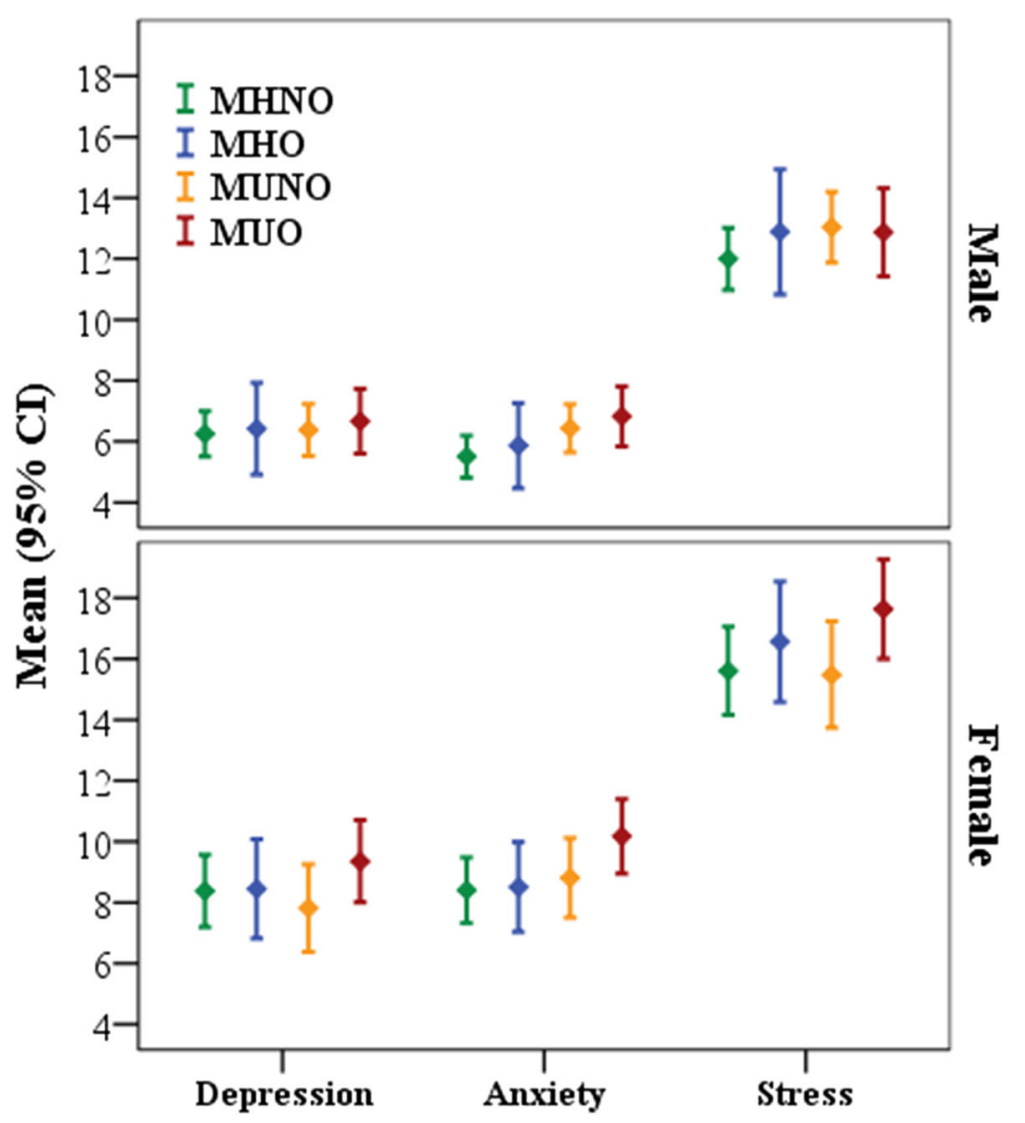

Fig. 1 The depression, anxiety, and stress means across sex-specific obesity phenotypes. The mean of DASS components is adjusted for age, marital status, education level, job status, and physical activity level

Moreover, indisputable effects of local culture on these global experiences can be seen in the findings; collectivism, one of the fundamental characteristics of Middle Eastern societies, helps prevent depression by social support but may result in increased anxiety. People in high collectivist cultures tend to attribute great significance to the social context, making them more exposed to anxiety [44]. In terms of sex differences, our findings indicated the importance of metabolic health status in increasing men's anxiety and stress levels, while neither obesity nor metabolic syndrome was solely associated with women's mental health conditions. One of the potential reasons for this sex difference could be the economic responsibility that men have in Iranian families [45], which could highlight the significance of men's health conditions.

The current findings revealed no significant association between obesity phenotypes and depression in both sexes. These results are consistent with the English Longitudinal Study of Ageing (ELSA) findings, which indicated that neither obesity nor poor metabolic status was associated with higher risks of depressive symptoms at over 2 years follow-up [28].
Accordingly, another longitudinal survey suggested that obesity was not a predictor of depression in Canadian women [13], and the same results have been observed among Mexican men [15]. Also, the lack of relationship between MetS and depression has been published among a sample of Turkish adults [20]. However, two systematic reviews confirmed the positive relationship between obesity and depression in American and Korean populations [11] and an increased risk of depression for MUO individuals [46]. Since the relation of obesity phenotypes with depression is multifactorial, the discrepancy in the outcomes could have a wide range of physical to psychosocial factors. Aside from gene-by-environment interaction [47], the underlying psychological factors seem to play a particular role in this regard. The increased prevalence of psychiatric morbidity, especially the depression in the treatment-seeking population, somehow reflects the difference in people's view of obesity [48]. Individuals with obesity who seek possible ways of losing their excessive weight experience obesity as a condition that needs to be changed. This point of view could be caused by psychological factors like 


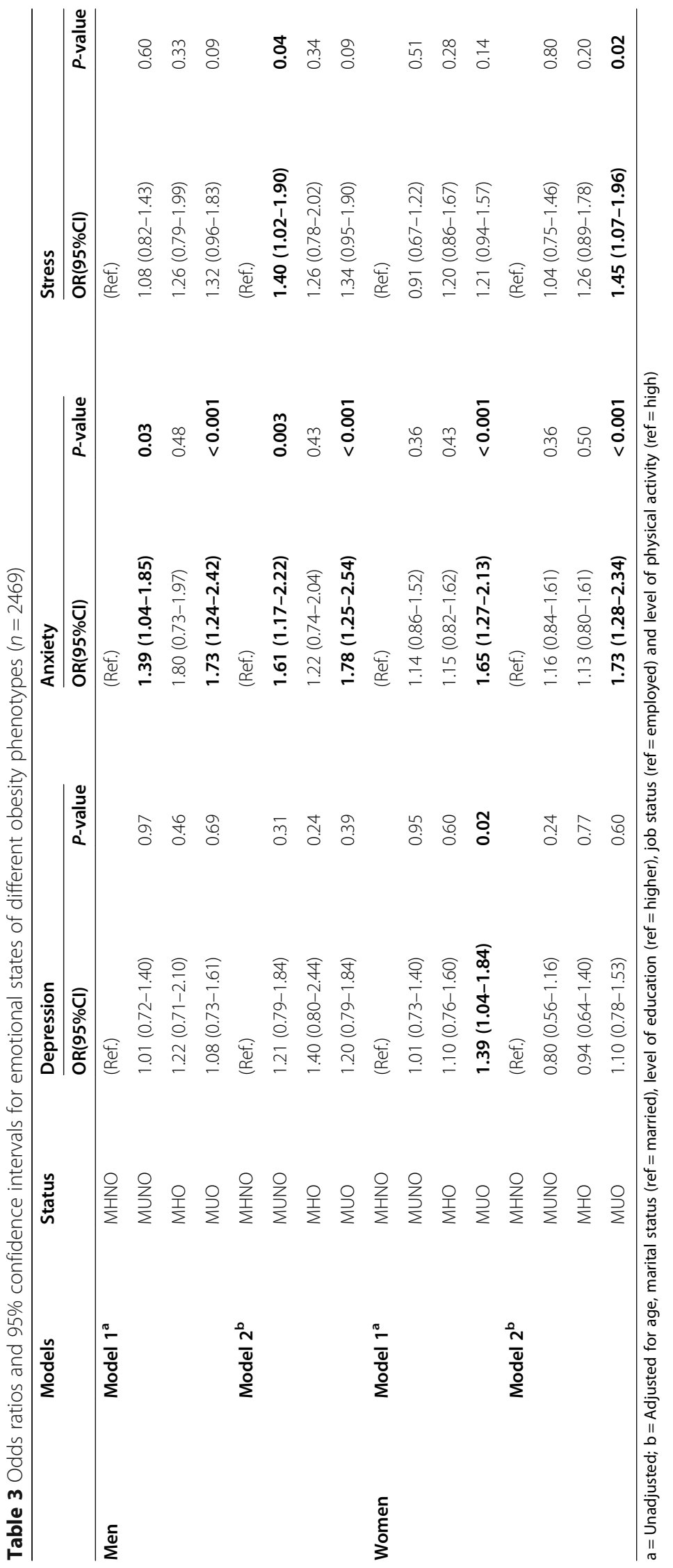


perceived body weight [49] and body dissatisfaction [50], which are strongly influenced by western media exposure and negative beliefs about obesity [51]. On the other hand, in some developing countries, obesity is considered a sign of health and wealth.; hence, since higher socio-economic groups are more likely to be obese, excessive weight does not have a significant psychological burden in these communities [51]. Additionally, Muslim countries like Iran have particular dress coding and clothing rules, which may lessen the importance of body shape and appearance and consequently lower psychological effects of weight status [14]. All these factors could make obesity a neutral factor in association with depression levels of the current study's population.

While the current study had strengths, there were some limitations. To the best of our knowledge, this is the first study to examine the synergic effects of weight and metabolic status on depression, anxiety, and stress among a large population of Tehranian adults. Nevertheless, due to the limitation of the cross-sectional design of the study, the causal relationship could not be established. It was also impracticable to adjust all the confounders due to the complicated essence of emotional distress; thus, the unmeasured variables could affect our findings. Additionally, these results can only be generalized to the Tehranian urban population.

\section{Conclusions}

In conclusion, men and women with various obesity phenotypes experience different anxiety and stress levels. While MUO women and all metabolically unhealthy men experienced more anxiety and stress levels than MHNO individuals, none of the obesity phenotypes were associated with depression. These valuable results on the psychological outcomes associated with different obesity phenotypes would be beneficial to recognize one of the somatic factors contributing to increased anxiety and stress symptoms in adults. These findings could draw the attention of physicians active in the field of metabolic disorders to assess patients' levels of anxiety and stress and require psychologists to determine the metabolic status of patients with high levels of anxiety and stress. Current results could also improve communities' public health by planning new strategies specific to each obesity phenotype.

\section{Abbreviations}

TLGS: Tehran lipid and glucose study; BMI: Body mass index; MetS: Metabolic syndrome; MHNO: Metabolically healthy non-obese; MHO: Metabolically healthy obese; MUNO: Metabolically unhealthy non-obese; MUO: Metabolically unhealthy obese (MUO); DASS_21: Depression, anxiety, and stress scale-21 items

\section{Supplementary Information}

The online version contains supplementary material available at https://doi. org/10.1186/s12888-021-03131-3.

\section{Additional file 1.}

Acknowledgments

Not applicable

Authors' contributions

P A and F M conceptualized the study. P A, F M, and L C participated in data curation. $L C$ carried out the statistical analysis. F M and P A designed the study. P A, F M, and L C contributed to interpretation of data. P A and F A were the project administrators. P A, F A and F $H$ supervised and revised the manuscript. F $M$ and $P$ A drafted the manuscript. P A, F M, L C, F H, and F A reviewed and edited the manuscript. All authors have read and approved the manuscript.

Funding

Not applicable.

Availability of data and materials

The datasets used and/or analyzed during the current study are available from the corresponding author on reasonable request.

\section{Declaration}

Ethics approval and consent to participate

This study was approved by the ethics committee of the Research Institute for Endocrine Sciences, Shahid Beheshti University of Medical Sciences. All the participants signed the written informed consent before data collection.

Consent for publication

Not applicable.

Competing interests

The authors declare that they have no competing interests in this section.

\section{Author details}

${ }^{1}$ Research Center for Social Determinants of Health, Research Institute for Endocrine Sciences, Shahid Beheshti University of Medical Sciences, P.O.Box: 19395-4763, Tehran, Iran. ²Department of Epidemiology and Biostatistics, Research Institute for Endocrine Sciences, Shahid Beheshti University of Medical Sciences, Tehran, Iran. ${ }^{3}$ Department of Psychiatry, Taleghani Hospital Research Development Committee, Shahid Beheshti University of Medical Sciences, Tehran, Iran. ${ }^{4}$ Obesity Research Center, Research Institute for Endocrine Sciences, Shahid Beheshti University of Medical Sciences, Tehran, Iran. ${ }^{5}$ Endocrine Research Center, Research Institute for Endocrine Sciences, Shahid Beheshti University of Medical Sciences, Tehran, Iran.

Received: 25 July 2020 Accepted: 21 February 2021

Published online: 04 March 2021

\section{References}

1. Organization WH. Obesity and overweight fact sheet. 2016. Department of Sustainable Development and Healthy Environments Available from: http:// www searo who int/entity/noncommunicable_diseases/media/non_ communicable_diseases_obesity_fs pdf accessed June10. 2018.

2. Rahmani A, Sayehmiri K, Asadollahi K, Sarokhani D, Islami F, Sarokhani M. Investigation of the prevalence of obesity in Iran: a systematic review and meta-analysis study. Acta Medica Iranica. 2015:596-607.

3. Apovian CM. Obesity: definition, comorbidities, causes, and burden. Am J Manag Care. 2016;22(7 Suppl):s176-85.

4. O'Neill S, O'Driscoll L. Metabolic syndrome: a closer look at the growing epidemic and its associated pathologies. Obes Rev. 2015;16(1):1-12.

5. Pajunen $\mathrm{P}$, Kotronen A, Korpi-Hyövälti E, Keinänen-Kiukaanniemi S, Oksa H, Niskanen L, et al. Metabolically healthy and unhealthy obesity phenotypes in the general population: the FIN-D2D survey. BMC Public Health. 2011; 11(1):754. 
6. Penninx BW, Lange SM. Metabolic syndrome in psychiatric patients: overview, mechanisms, and implications. Dialogues Clin Neurosci. 2018; 20(1):63.

7. Kendrick T, Pilling S. Common mental health disorders-identification and pathways to care: NICE clinical guideline. Br J Gen Pract. 2012;62(594):47-9.

8. Baxter A, Vos T, Scott K, Ferrari A, Whiteford H. The global burden of anxiety disorders in 2010. Psychol Med. 2014;44(11):2363-74.

9. Malhotra N, Kulhara P, Chakrabarti S, Grover S. Lifestyle related factors \& impact of metabolic syndrome on quality of life, level of functioning \& selfesteem in patients with bipolar disorder \& schizophrenia. Indian J Med Res. 2016;143(4):434

10. Valizadeh R, Sarokhani D, Sarokhani M, Sayehmiri K, Ostovar R, Angh P, Malekzadeh M. A study of prevalence of anxiety in Iran: systematic review and meta-analysis. Der Pharma Chemica. 2016;8(21):48-57. https://doi.org/1 0.15171/jarcm.2016.020.

11. Rajan T, Menon V. Psychiatric disorders and obesity: a review of association studies. J Postgrad Med. 2017;63(3):182

12. Amiri S, Behnezhad S. Obesity and anxiety symptoms: A systematic review and meta-analysis. Neuropsychiatrie. 2019;33(2):72-89.

13. Gariepy G, Wang J, Lesage AD, Schmitz N. The longitudinal association from obesity to depression: results from the 12-year National Population Health Survey. Obesity. 2010;18(5):1033-8.

14. Askari J, Hassanbeigi A, Khosravi HM, Malek M, Hassanbeigi D, Pourmovahed $Z$, et al. The relationship between obesity and depression. Procedia Soc Behav Sci. 2013:84:796-800.

15. Zavala GA, Kolovos S, Chiarotto A, Bosmans JE, Campos-Ponce M, Rosado $J$, et al. Association between obesity and depressive symptoms in Mexican population. Soc Psychiatry Psychiatr Epidemiol. 2018;53(6):639-46.

16. Rahmani J, Kord Varkaneh H, Dorosty A-R. The relationship between general and central obesity with anxiety among Iranian young men. J Nutr Food Secur. 2018;3(1):4-12.

17. Minkwitz J, Scheipl F, Cartwright L, Campbell IC, Chittka T, Thormann J, et al. Why some obese people become depressed whilst others do not: exploring links between cognitive reactivity, depression and obesity. Psychol Health Med. 2019;24(3):362-73.

18. Crisp AH, McGuiness B. Jolly fat: relation between obesity and psychoneurosis in general population. Br Med J. 1976;1(6000):7-9.

19. Takeuchi T, Nakao M, Nomura K, Yano E. Association of metabolic syndrome with depression and anxiety in Japanese men. Diabetes Metab. 2009;35(1): 32-6.

20. Demirci H, Cinar Y, Bilgel N. Metabolic syndrome and depressive symptoms in a primary health care setting in Turkey. Bull Clin Psychopharmacol. 2011; 21(1):49-57.

21. Gheshlagh RG, Parizad N, Sayehmiri K. The relationship between depression and metabolic syndrome: systematic review and meta-analysis study. Iran Red Crescent Med J. 2016;18(6):e26523. https://doi.org/10.5812/ircmj.26523.

22. Bagherniya M, Khayyatzadeh SS, Avan A, Safarian M, Nematy M, Ferns GA, et al. Metabolic syndrome and its components are related to psychological disorders: a population based study. Diabetes Metab Syndr Clin Res Rev. 2017;11:S561-S6.

23. Tomiyama AJ. Stress and obesity. Annu Rev Psychol. 2019;70:703-18.

24. Tenk J, Mátrai P, Hegyi P, Rostás I, Garami A, Szabó I, et al. Perceived stress correlates with visceral obesity and lipid parameters of the metabolic syndrome: a systematic review and meta-analysis. Psychoneuroendocrinology. 2018:95:63-73.

25. Jokela M, Hamer M, Singh-Manoux A, Batty G, Kivimäki M. Association of metabolically healthy obesity with depressive symptoms: pooled analysis of eight studies. Mol Psychiatry. 2014;19(8):910-4.

26. Phillips CM, Perry IJ. Depressive symptoms, anxiety and well-being among metabolic health obese subtypes. Psychoneuroendocrinology. 2015:62:47-53.

27. Hinnouho G-M, Singh-Manoux A, Gueguen A, Matta J, Lemogne C, Goldberg $M$, et al. Metabolically healthy obesity and depressive symptoms: 16-year follow-up of the Gazel cohort study. PLoS One. 2017;12(4):e0174678.

28. Slater N, Rowley C, Venables RH, White S, Frisher M. Evaluating associations between metabolic health, obesity and depressive symptoms: a prospective analysis of data from the English Longitudinal Study of Ageing (ELSA) with a 2-year follow-up. BMJ Open. 2018;8(12):e025394. https://doi.org/10.1136/ bmjopen-2018-025394.

29. Yosaee S, Djafarian K, Esteghamati A, Motevalian A, Shidfar F, Tehrani-Doost $M$, et al. Depressive symptoms among metabolically healthy and unhealthy overweight/obese individuals: a comparative study. Med J Islam Repub Iran. 2018;32:95.

30. Azizi F, Ghanbarian A, Momenan AA, Hadaegh F, Mirmiran P, Hedayati M, et al. Prevention of non-communicable disease in a population in nutrition transition: Tehran lipid and glucose study phase II. Trials. 2009;10(1):5.

31. Hadaegh F, Bozorgmanesh MR, Ghasemi A, Harati H, Saadat N, Azizi F. High prevalence of undiagnosed diabetes and abnormal glucose tolerance in the Iranian urban population: Tehran lipid and glucose study. BMC Public Health. 2008:8(1):176.

32. Azizi F, Khalili D, Aghajani H, ESTEGHAMATI A, Hosseinpanah F, DELAVARI A, et al. Appropriate waist circumference cut-off points among Iranian adults: the first report of the Iranian National Committee of obesity. 2010.

33. AZIZI F, Hadaegh F, KHALILI D, Esteghamati A, HOSSEIN PF, Delavari A, et al Appropriate definition of metabolic syndrome among Iranian adults: report of the Iranian National Committee of obesity. 2010.

34. Momenan AA, Delshad M, Sarbazi N, REZAEI GN, Ghanbarian A, AZIZI F. Reliability and validity of the modifiable activity questionnaire (MAQ) in an Iranian urban adult population. 2012.

35. Asghari A, Saed F, Dibajnia P. Psychometric properties of the depression anxiety stress Scales-21 (DASS-21) in a non-clinical Iranian sample. Int J Psychol. 2008;2(2):82-102.

36. Lovibond S, Lovibond PF. Psychology Foundation of Australia.(1995). Manual for the depression anxiety stress scales.

37. Benjamini Y, Hochberg Y. Controlling the false discovery rate: a practical and powerful approach to multiple testing. J R Stat Soc Ser B Methodol. 1995:57(1):289-300.

38. Boyd A, Van de Velde S, Vilagut G, De Graaf R, Florescu S, Alonso J, et al. Gender differences in mental disorders and suicidality in Europe: results from a large cross-sectional population-based study. J Affect Disord. 2015; 173:245-54.

39. Li SH, Graham BM. Why are women so vulnerable to anxiety, trauma-related and stress-related disorders? The potential role of sex hormones. Lancet Psychiatry. 2017:4(1):73-82

40. Johnson DP, Whisman MA. Gender differences in rumination: a metaanalysis. Personal Individ Differ. 2013;55(4):367-74.

41. Kuehner C. Why is depression more common among women than among men? Lancet Psychiatry. 2017:4(2):146-58.

42. Küçük S. Being a woman in Turkey and in the Middle East. Turkish Policy Quartely. 2013;11(4):23-43.

43. Khan S, Khan RA. Chronic stress leads to anxiety and depression. Ann Psychiatry Ment Health. 2017;5(1):1091.

44. Chavira DA, Ponting C, Lewis-Fernández R. Cultural and social aspects of anxiety disorders, vol. 59. Washington, DC: The American Psychiatric Association Publishing Textbook of Anxiety, Trauma, and OCD-Related Disorders; 2020

45. Esfahani HS, Shajari P. Gender, education, family structure, and the allocation of labor in Iran. Middle East Dev J. 2012;4(02):1250008.

46. Malmir H, Mirzababaei A, Moradi S, Rezaei S, Mirzaei K, Dadfarma A. Metabolically healthy status and BMI in relation to depression: a systematic review of observational studies. Diabetes Metab Syndr Clin Res Rev. 2019.

47. McCaffery JM, Doyle CY. 7 genex environment interaction in obesity: the contribution of randomized clinical trials. Statistical Approaches to Gene X Environment Interactions for Complex Phenotypes 2016:117.

48. Vaidya V. Psychosocial aspects of obesity. Health and Treatment strategies in obesity. 27. Basel: Karger Publishers; 2006. p. 73-85. https://doi.org/10.11 59/000090965.

49. Paulitsch RG, Demenech LM, Dumith SC. Association of depression and obesity is mediated by weight perception. J Health Psychol. 2020 1359105319897778. https://doi.org/10.1177/1359105319897778.

50. Richard A, Rohrmann S, Lohse T, Eichholzer M. Is body weight dissatisfaction a predictor of depression independent of body mass index, sex and age? Results of a cross-sectional study. BMC Public Health. 2016;16(1):863.

51. Khaled SM, Shockley B, Qutteina Y, Kimmel L, Le KT. Testing Western media icons influence on Arab Women's body size and shape ideals: an experimental approach. Soc Sci. 2018;7(9):142.

\section{Publisher's Note}

Springer Nature remains neutral with regard to jurisdictional claims in published maps and institutional affiliations. 